\title{
Hospital admissions for asthma and chronic obstructive airways disease in East London hospitals and proximity of residence to main roads
}

\author{
S E Morris, R C Sale, J C Wakefield, S Falconer, P Elliott, B J Boucher
}

We describe a matched case-control study investigating the association between respiratory illness and proximity of residence to main roads. The study was carried out in response to previously inconsistent reports on this question $^{1-3}$; the null hypothesis was of no association of traffic related air pollution to respiratory illness, hospital admissions for asthma and chronic obstructive airways disease. The study focused on Tower Hamlets, East London where hospital admissions for asthma are $80 \%$ above national rates. ${ }^{12}$

\section{Department of \\ Epidemiology and \\ Public Health, \\ Imperial College \\ School of Medicine, St \\ Mary's Campus, \\ London W2 1PG \\ $S$ E Morris \\ J C Wakefield \\ $S$ Falconer \\ P Elliott}

\section{Department of}

General and

Emergency Medicine,

Royal London

Hospital, London

R C Sale

B J Boucher

Correspondence to:

Professor P Elliott

Accepted for publication 23 June 1999

\section{Methods}

The cases were extracted from the medical summaries of sequential emergency medical admissions through the casualty departments at The Royal London Hospitals in 1991-1992. Patient records with a diagnosis of asthma or chronic obstructive airways disease, and resident in Tower Hamlets, were identified. Individually matched controls (also obtained from emergency medical admissions through the casualty departments) with a non-chest related illness were selected based on sex, year of admission and consultant team. The selection method of these controls precludes surgical, orthopaedic or trauma cases. Overall, $82 \%$ of the pairs were matched to within five years, the remainder to within 10 years. For each case and control, address, ethnic group, whether there had been treatment in intensive therapy, and whether there had been any re-admission

Table 1 Summary of covariates and unadjusted estimated odds ratios for asthma and chronic airways disease cases and controls. Figures in parentheses are percentages. High values of the deprivation score indicate the most deprived areas (the Carstairs index has mean zero across England and Wales)

\begin{tabular}{lllll}
\hline Covariate & Asthma cases & Asthma controls & COAD cases & COAD controls \\
\hline $\begin{array}{l}\text { Sex } \\
\text { Male }\end{array}$ & $23(18.4)$ & $23(18.4)$ & $75(60.5)$ & $75(60.5)$ \\
Female & $102(81.6)$ & $102(81.6)$ & $49(39.5)$ & $49(39.5)$ \\
Age (y) & & & & \\
$15-40$ & $73(58.4)$ & $70(56.0)$ & $2(1.6)$ & $0(0)$ \\
$41-65$ & $41(32.8)$ & $40(32.0)$ & $45(36.3)$ & $54(43.5)$ \\
$66-90$ & $11(8.8)$ & $13(10.4)$ & $77(62.1)$ & $70(56.5)$ \\
Ethnic group & & & & \\
White & $65(52.0)$ & $71(56.8)$ & $85(68.5)$ & $84(67.7)$ \\
Asian & $28(22.4)$ & $24(19.2)$ & $19(15.3)$ & $21(16.9)$ \\
Black & $2(1.6)$ & $6(4.8)$ & $0(0)$ & $0(0)$ \\
Other & $25(20.0)$ & $15(12.0)$ & $13(10.5)$ & $13(10.5)$ \\
Unknown & $5(4.0)$ & $9(7.2)$ & $6(4.8)$ & $6(4.8)$ \\
Deprivation & & & & \\
Median & 6.6 & 5.98 & 6.21 & 6.13 \\
(1st, 3rd quartiles $)$ & $(4.4,11.1)$ & $(4.4,9.7)$ & $(4.7,10.6)$ & $(4.3,9.9)$ \\
Distance & & & & \\
$\leqslant 150 \mathrm{~m}$ & 84 & 91 & 85 & 87 \\
$>150 \mathrm{~m}$ & 41 & 34 & Estimated odds ratio $=0.94$ \\
& Estimated odds ratio $=0.78$ & $(95 \%$ CI $0.57,1.54)$ \\
\hline
\end{tabular}

during that year, were extracted. Smoking data were too incomplete to be used.

Using the Address-point software the addresses of cases and controls were expressed as a grid reference for all but eight participants. This software allows each postal address to be located to the "front door" at ground level (accurate to $0.1 \mathrm{~m}$ ). Truncated grid references were obtained for six of the eight missing people; for one, the grid reference of a neighbouring house, and for the remainder, a different flat number within the same block of flats was used. The Carstairs deprivation index was used to classify each case and control using the census enumeration district/postcode link. Eleven case-control pairs were removed from the analysis because the Carstairs index was unavailable for either the case or the control. This removed one suspect address, and left 125 asthma and 124 chronic obstructive airways disease pairs.

Bartholomew's road directory and a Geographic Information System were used to calculate the distance of each address to the nearest "main" road (motorway, primary, A or B road).

Distance from road was considered as both a discrete two level factor ( $\leqslant 150 \mathrm{~m},>150 \mathrm{~m}$ ) and as a continuous variable. For the continuous analysis a model with a smooth monotonic relation between risk and distance was assumed, and was compared with the null model of no relation. ${ }^{4}$ For both models, because of the matched design, a conditional likelihood approach was taken, with adjustment made for age and deprivation, the former to account for the non-exact nature of the matching.

\section{Results}

Six asthma cases (11 chronic obstructive airways disease cases) required intensive therapy, and nine asthma cases (11) were readmitted in the study period. Table 1 gives data on covariates and distance from roads. Cases and controls for asthma were relatively well matched. Ninety one per cent of the addresses of cases/controls fell within the most deprived quintile of enumeration districts for England and Wales. The unadjusted odds ratio for living within $150 \mathrm{~m}$ of a main road was $0.78(95 \%$ confidence intervals $0.46,1.32$ ) and with adjustment for age and deprivation it was 0.86 $(0.50,1.48)$. With distance as a continuous variable the monotonic distance/risk model provided no improvement over the null model.

For chronic obstructive airways disease the unadjusted odds ratio was $0.94(0.57,1.54)$ and with adjustment for age and deprivation it 
was $0.97(0.58,1.60)$. The monotonic distance/risk model provided a significant improvement on the null model $(\mathrm{p}=0.024)$. The estimated odds ratio at roadside was 5.6 falling to unity by $50 \mathrm{~m}$, indicating an association at short distances, and explaining why the discrete analysis did not detect an effect. Further analysis on three levels $(<50 \mathrm{~m}$, 50-150 m, >150 m) showed no significant trend. When the case-control pairs with non-exact addresses were removed the results were unchanged for asthma but for chronic obstructive airways disease (four pairs) the $\mathrm{p}$ value was increased to 0.056 .

\section{Discussion}

For asthma the odds ratios were not significantly different from zero in agreement with results of a study in Tower Hamlets of GP treatment and diagnosis of asthma. ${ }^{3}$ For chronic obstructive airways disease, while the discrete analysis provided no evidence of association, the continuous analysis, which has the advantage of estimating the extent of any effect, gave weak evidence for a highly localised (within $50 \mathrm{~m}$ ) association between risk and distance of residence from a main road. This result was, however, sensitive to the inclusion of four cases without full address information. Cases within two of these pairs were located within $50 \mathrm{~m}$ of a main road.

No clear picture emerges from this analysis. Although a highly localised effect of traffic related pollution on respiratory health is plausible (as pollutants are increased above background up to that distance ${ }^{5}$ ), the lack of smoking data presents a major difficulty limiting interpretation. Other problems include possible exposure mis-classification, as exposure to road traffic was estimated at place of residence only and no allowance could be made for height of residence above ground, nor daily activity patterns. Place of residence may be a poor indicator of personal exposure to traffic related pollutants. In addition, diagnosis relied on routine recording in hospital notes, and milder cases not requiring admission would be missed. The study focused on adults. Children may be more susceptible: positive associations of respiratory illness with road traffic have been reported in some studies, ${ }^{12}$ but not others. ${ }^{3}$ Future studies need to obtain improved estimates of exposure at residence, for example, through dispersion modelling. ${ }^{5}$ They should preferably include (a) questionnaire data to give information on smoking, indoor sources, etc, and time activity patterns; and (b) improved health outcome data, possibly including lung function measurements.

The study was carried out with the approval of the District Ethical Committee. We thank the Consultant Physicians Dr N C Barnes, Dr M Chaput de Saintonge, Professor R D Cohen, Dr J Cunningham, Dr D D Gibbs, Dr M Glynn, Dr D T D Hughes, Dr F P Marsh, Professor D W Vere and Dr D Empey for allowing their patient records to be analysed and Professor Peter Diggle of the Department of Statistics at Lancaster University for useful discussions on the continuous risk modelling. This work was supported by ESRC grant number R000237819. The 1991 census data was provided with the support of the ESRC. Digital map data copyright Bartholomew. This work was supported, in part, by an equipment grant from the Wellcome Trust and is based on data supplied by Ordnance Survey and these data remain the property of the Crown and copyright of HMSO and Royal Mail.

Conflicts of interest: none.

S E Morris carried out the statistical analysis and helped draft the paper; R C Sale abstracted the data from medical records and approved the paper; J C Wakefield oversaw the statistical and approved the paper; J C Wakefield oversaw the statistical analysis and helped draft the paper; $S$ Falconer carried out the GIS analysis; P Elliott helped initiate the study and draft the
paper; B J Boucher initiated the study and helped draft the paper;
paper.

1 Wist M, Reitmar P, Dold S, Wuiff A, Nicolai T, von Loeffelholtz-Colberg EF, et al. Road traffic and adverse
effects on respiratory health in children. BMF 1993;307:596-600.

2 Edwards J, Walters S, Griffiths RC. Hospital admissions for asthma in pre-school children: relationship to major roads
in Birmingham UK. Arch Environ Health 1994;49:223-7.

3 Livingstone AE, Shaddick G, Grundy C, Elliott P. Do people living near inner city main roads have more asthma
plingstone AE, Shaddick $\mathrm{G}$, Grundy needing treatment? Case-control study. BMF 1996;311: $676-7$.

4 Diggle P, Rowlingson B. A conditional approach to point process modelling of raised incidence. Fournal of the Royal Statistical Society, Series A 1994; 157:433-40.

5 Design manual for roads and bridges: vol 11 Environmental Assessment. Department of Transport, The Scottish Office Industrial Department, The Welsh Office, Department of Environment for Northern Ireland:1993. 